\title{
Mesenchymal stem cells in the pathogenesis and treatment of bronchopulmonary dysplasia: a clinical review
}

\author{
Ann A. Simones ${ }^{1}$, Daniel J. Beisang ${ }^{1}$, Angela Panoskaltsis-Mortari ${ }^{1}$ and Kari D. Roberts ${ }^{1}$
}

Advances in neonatal medicine have led to increased survival of infants born at the limits of viability, resulting in an increased incidence of bronchopulmonary dysplasia (BPD). $\mathrm{BPD}$ is a chronic lung disease of premature infants characterized by the arrest of alveolarization, fibroblast activation, and inflammation. BPD leads to significant morbidity and mortality in the neonatal period and is one of the leading causes of chronic lung disease in children. The past decade has brought a surge of trials investigating cellular therapies for the treatment of pulmonary diseases. Mesenchymal stem cells (MSCs) are of particular interest because of their ease of isolation, low immunogenicity, and antiinflammatory and reparative properties. Clinical trials of MSCs have demonstrated short-term safety and tolerability; however, studies have also shown populations of MSCs with adverse pro-inflammatory and myofibroblastic characteristics. Cell-based therapies may represent the next breakthrough therapy for the treatment of BPD, however, there remain barriers to implementation as well as gaps in knowledge of the role of endogenous MSCs in the pathogenesis of BPD. Concurrent high-quality basic science, translational, and clinical studies investigating the fundamental pathophysiology underlying BPD, therapeutic mechanisms of exogenous MSCs, and logistics of translating cellular therapies will be important areas of future research.

A dvances in neonatal medicine have resulted in increased survival of infants born at the limits of viability and fostered a trend toward resuscitation of infants born at earlier gestational ages-in some centers as early as 22-weeks gestation. Due to the extreme immaturity of lung development at these early gestations, respiratory sequelae remain a significant cause of mortality and long-term morbidity. Bronchopulmonary dysplasia (BPD), a chronic lung disease of premature infants, was originally described in 1967 as a fibrotic lung injury resulting from prolonged mechanical ventilation and oxygen exposure (1). Over the past several decades, discovery of surfactant, implementation of gentle ventilation strategies, and effective non-invasive ventilation devices have resulted in a reduction of prolonged mechanical ventilation and oxygen exposure. Despite these advances, the incidence of BPD has continued to rise due to the emergence of a "new BPD". This "new BPD" is characterized by tissue simplification and arrest of alveolarization rather than the fibrosis and inflammation associated with the "old BPD" $(2,3)$.

Of infants born between 22- and 27-weeks gestation, 45\% develop BPD, accounting for $>10,000$ new cases in the United States each year (4). Investigation of targeted therapies such as caffeine, vitamin A, anti-inflammatories, and steroids has shown either minimal treatment effect or clinical use has been limited by significant side effects (5). With increased survival of infants at the limits of viability and the incidence of $\mathrm{BPD}$ on the rise, the search for innovative therapies for BPD has become an area of heightened interest.

Emerging research in the field of stem cell biology has generated enthusiasm that stem cells may prove to be the next breakthrough therapy for BPD. It is known that mesenchymal induction is critical to alveolarization, and that mesenchymal stem (stromal) cells (MSCs) play a key role in lung growth and repair (6). There is extensive pre-clinical evidence that exogenously administered MSCs ameliorate lung injury in experimental BPD models (7-11) and early clinical trials in human neonates are currently underway $(12,13)$. However, while some studies have shown growth and antiinflammatory properties, other studies have shown paradoxical adverse pro-inflammatory and myofibroblastic characteristics (14). This review will focus on pre-clinical and clinical research that has contributed to current knowledge of the role of MSCs in lung disease as well as an overview of clinical trials investigating the use of therapeutic MSCs for neonatal, pediatric, and adult pulmonary diseases, with a focus on BPD.

\section{MESENCHYMAL STEM CELLS}

MSCs are multipotent progenitor cells that reside within all tissues in the body (15). This cell population plays a critical role in directing developmental programing and cell response to tissue injury (6). MSCs are defined by three criteria including (i) adherence to plastic in cell culture dish, (ii) expression of MSC-defining cell surface markers including CD73, CD90, and CD105, and (iii) tri-lineage potential 
including ability to differentiate into osteocytes, adipocytes, and chondrocytes (16).

Determining how and where exogenously administered MSCs work has been the focus of considerable research. Despite initial results suggesting exogenous MSCs function through engraftment, subsequent findings have shown that exogenous MSCs are likely functioning through paracrine signaling. Data from animal models have identified few engrafted MSCs in recipient lungs, and administration of both MSCs as well as MSC-conditioned media has been shown to confer long-term improvement in lung function and architecture $(17,18)$. Additionally, bronchoalveolar stem cells (BASCs), progenitor cells found in the distal lung epithelium, are found in greater numbers in the lungs of animals treated with MSCs (19). These facts support the hypothesis that MSCs are promoting endogenous repair processes through paracrine signaling as opposed to replacing host MSCs $(17,20)$. Although speculative, it is likely that native lung MSC biology is impacted by exogenously delivered MSCs, and an interesting question involves the mechanism by which this interaction may be occurring. Exogenous MSCs may directly interact with resident MSCs to alter their biology, or alternatively, paracrine signaling from exogenous MSCs may occur indirectly via cells of the distal lung epithelium and alveolar cellular constituents, which are in turn impacting native MSC biology.

It is proposed that the paracrine effect of MSCs is mediated through secretion of a variety of bioactive substances including signaling peptides (interleukin-6, interleukin-8, and vascular endothelial growth factor), extracellular matrix proteins (collagen and elastin), and exosomes (6,21-23). Exosomes are membrane-bound packages of cellular products including proteins, microRNAs, and mRNA species that are taken up by near-by cells and influence gene expression patterns and cellular biology (24). Exosomes are increasingly recognized as important mediators of cell-to-cell communication. Target cells of MSC-derived exosomes include fibroblasts, MSCs, epithelial cells, and immune cells (24). It is partially through this exosome-mediated mechanism that MSCs exert their anti-inflammatory effect in vitro $(24,25)$.

The role of exosomes in the treatment of lung disease is an area of active pre-clinical study. In an animal model of chronic hypoxia, intravenous MSC-derived exosomes were shown to ameliorate pulmonary hypertension and right ventricular hypertrophy, suggesting that exosomes may have therapeutic potential in the prevention and repair of lung disease (26). Additionally, preliminary findings from a study of intratracheally delivered MSCs vs. MSC-derived exosomes showed that exosomes were more effective than whole MSCs at improving lung compliance and survival in hyperoxiainduced lung disease in rats (27).

These data on cell-free preparations raise interest in their potential clinical application for BPD prevention and treatment. Cell-free preparations have potential advantages over cell-based therapies including the ability to avoid theoretical complications of progenitor cell delivery.
Exosomes may also serve as mechanisms for intercellular delivery of molecular cargo with the potential to deliver pharmaceutical products in an efficient manner (28). Despite these apparent advantages, significant knowledge gaps exist and additional pre-clinical data are required before transitioning to clinical trials.

\section{LUNG-RESIDENT MESENCHYMAL STEM CELLS}

While MSCs play a key role in growth and repair, several studies indicate that under certain conditions, MSCs may contribute to the pathogenesis of pulmonary disease (29). Lung-resident MSCs (L-MSCs) play a critical role in alveolarization through conduct of the tightly regulated processes of alveolar septation and vascularization (30). Disruption of this process leads to simplified alveolar architecture and abnormal pulmonary vasculature contributing to pulmonary hypertension-a key feature of "new BPD" (3). L-MSCs also play an important role in directing injuryrepair responses in the lung. L-MSCs have a dichotomous response potential including normal healing on the one hand and a fibrotic response on the other (Figure 1); when this balance is disrupted during injury repair, fibrotic lung disease results (6). The multi-lineage potential of MSCs and the multi-faceted role of these cells during development and wound healing necessitates a robust and intricate molecular signaling network to direct the function of these cells to carry out the appropriate function under the appropriate circumstances. Much work has been done to unravel these pathways during human development and many key signaling compounds are now better understood. It is well established that signaling via transforming growth factor-beta (TGF- $\beta$ ), platelet-derived growth factor (PDGF), fibroblast growth factor (FGF), and Wnt/beta-catenin pathways, among others, are important in determining the cellular phenotype of MSCs (31-34). In preterm infants, isolation of L- MSCs from tracheal aspirates (35) and elevated levels of TGF- $\beta$ in blood and tracheal aspirates $(36,37)$ were correlated with later development of BPD. It has also been shown that L-MSCs from neonatal tracheal aspirates compared to cord-bloodderived MSCs exhibited altered levels of expression for multiple genes involved in development, growth factors, extracellular matrix, inflammatory mediators, and lipid metabolism (38). In addition, these L-MSCs acquired a myofibroblastic phenotype in response to TGF- $\beta 1$, a response that was not seen in bone marrow-derived MSCs (BM-MSCs), highlighting the distinct properties of MSCs from differing sources (32). Evidence suggesting that MSCs exist in at least two different phenotypes, one of which has the capacity to promote inflammation and fibrosis (23), raises concern for potential transformation of exogenously administered MSCs to a detrimental phenotype. Thus, a better understanding of this heterogeneity and the microenvironmental stimuli that promote divergent phenotypes is important as this technology transitions from the bench to the bedside. 


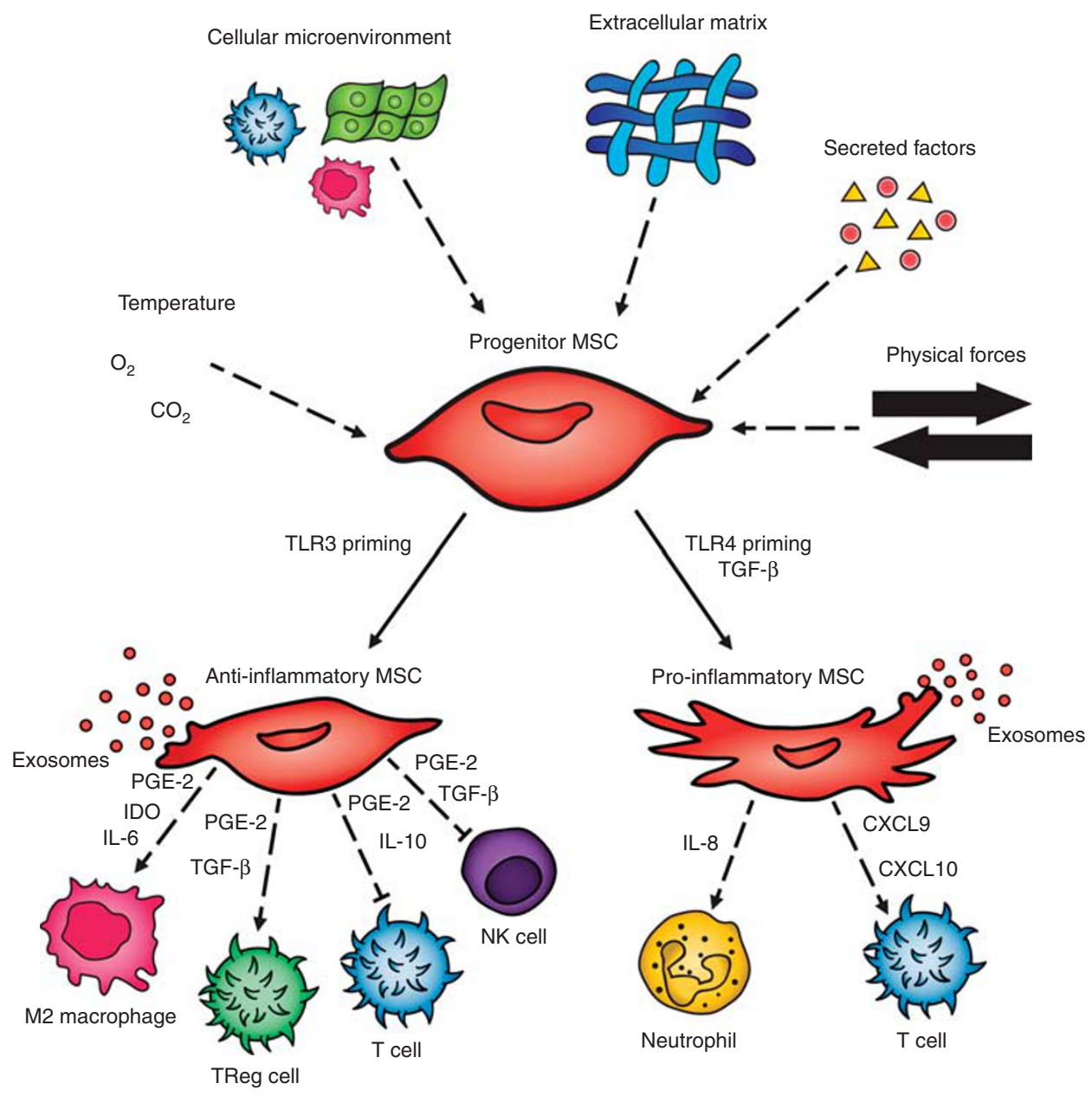

Figure 1. Mesenchymal stem cell (MSC) function is regulated by the complex interplay of numerous microenvironmental stimuli, including local cellcell interactions, physical stimuli from the extracellular matrix and mechanical stress, and chemical signals from circulating factors, oxygen tension, and acid-base status (64). Under certain environmental stimuli, some populations of MSCs transform into divergent phenotypes (6). Toll-like receptor3 (TLR-3) priming has been shown to induce an anti-inflammatory phenotype (14). Anti-inflammatory MSCs regulate a variety of inflammatory cells through the secretion of exosomes and bioactive substances such as prostaglandin E-2 (PGE-2), transforming growth factor- $\beta$ (TGF- $\beta$ ), interleukin- 6 (IL-6), interleukin-10 (IL-10), and indoleamine 2,3-dioxygenase (IDO) (23). TGF- $\beta$ and Toll-like receptor-4 (TLR-4) induce a pro-inflammatory phenotype $(14,32)$, which causes activation of pro-inflammatory cells through the secretion of exosomes and bioactive substances such as interleukin-8 (IL-8), chemokine ligand 9 (CXCL9), and chemokine ligand 10 (CXCL10) (23).

\section{OPTIMAL ROUTE, DOSE, AND TIMING}

Pre-clinical and clinical studies have focused on the optimal route, dosing, and timing for MSC delivery. In animal models of BPD, MSCs have been delivered systemically (intravenous (IV) or intraperitoneal (IP)) as well as intratracheal (IT). In a pre-clinical study of hyperoxia-induced lung injury in neonatal rats, comparison of systemic vs. IT MSC administration found that IT administration was more efficacious at reversing lung injury at a four-fold lower dose of MSCs (7). The ability to achieve equal efficacy with lower required cell numbers makes IT administration an attractive route of administration moving into the clinical realm.

In addition to understanding the route of administration, the dose of cells is an important preclinical question as well. Multiple studies have investigated the efficacy of IT MSC delivery for BPD, and these have typically included doses of $\sim 10^{5}$ cells per rat administered anywhere from P3 to P10
$(7,9-11,17)$. Direct comparison of doses of $5 \times 10^{3}, 5 \times 10^{4}$, and $5 \times 10^{5}$ cells per rat administered on P5 in the hyperoxiainduced lung injury model have been performed, with a dosedependent effect on lung architecture, survival, inflammatory makers, and collagen production, with $5 \times 10^{5}$ cells/rat being the most efficacious dose (11). Note that with P5 rats weighing on the order of $10 \mathrm{~g}$, this dose is on the order of $10^{7}$ cells $/ \mathrm{kg}$. As discussed in detail later in this review, clinical studies in humans investigating the appropriate dose of IT MSCs for treatment of BPD are limited to a phase I dose escalation trial that included doses of $1 \times 10^{7}$ and $2 \times 10^{7}$ cells/ $\mathrm{kg}$. Both doses appeared to be safe without increased shortterm or long-term adverse events $(12,13)$.

Finally, optimal timing of treatment is another important question that preclinical studies have sought to answer. Our own group investigated the optimal timing of MSC delivery in a murine model of bronchiolitis obliterans following bone 
marrow transplant. This study showed similar improvements in inflammation, lung mechanics, and histology with both early ( 2 weeks post transplant) and late (4 weeks post transplant) administration of IT MSCs (39). Conversely, a rat hyperoxia model of BPD found that early, but not late, administration of MSCs was efficacious suggesting that there may be a critical window during the pathogenesis of BPD within which MSC administration must occur for optimal effect (10).

\section{WHAT CAN WE LEARN FROM CLINICAL TRIALS OF MSCS FOR ADULT LUNG DISEASES?}

The past decade has brought a surge of clinical trials investigating the safety and efficacy of MSCs for adult diseases including pulmonary diseases, myocardial infarction, stroke, spinal cord injury, and graft-vs.-host disease. A metaanalysis published in 2012 sought to systematically evaluate the safety of this rapidly emerging new therapy (40). Evaluation of 36 clinical trials found no association of MSC therapy with significant adverse events, nor malignant transformation. Transient fever was the most commonly reported mild side effect of MSC infusion. One study reported an acute infusional hypersensitivity reaction in a single subject, however this was attributed to dimethylsulfoxide in the MSC preparation (41). The authors of this review cautiously concluded that IV MSC therapy appeared to be well-tolerated and safe in the short-term, however, ongoing follow-up of these subjects would be necessary before conclusions regarding long-term safety could be made.

Following the reassuring results of these early studies, the number of clinical trials utilizing MSC therapy has continued to increase. Currently, a query of "mesenchymal stem cell" on clinicaltrials.gov yields 745 results (42). Forty trials are specific to investigating MSCs in the treatment of adult and pediatric pulmonary diseases (Table 1 ).

To date, there are published outcomes from five completed phase 1 trials investigating the safety of MSCs for adult pulmonary diseases $(43-47)$. In combination, these trials have studied 101 patients with chronic obstructive pulmonary disease (COPD)/emphysema, acute respiratory distress syndrome (ARDS), and idiopathic pulmonary fibrosis (IPF). Administered cell dose ranged from $1 \times 10^{6}$ to $1 \times 10^{7}$ cells $/ \mathrm{kg}$ delivered intravenously as a single dose or as many as four repeated doses 1-4 weeks apart. No serious acute adverse events attributed to MSC therapy were reported in any of the studies. Though not powered to detect differences in outcomes between the groups, one study showed that patients with COPD and an elevated C-reactive protein (CRP) at baseline had a significant reduction in CRP level following MSC treatment (43). To address the concern for pro-fibrotic and tumorigenic potential of MSCs, two studies performed chest computerized tomography (CT) scans at either 3 and 6 months or 1 year post-MSC treatment $(44,48)$. No fibrosis or tumor formation was noted on CT in the MSC-treated groups in either of these studies. In addition, one study evaluated histology and gene expression changes in lung tissue in patients treated with MSCs by performing lung volume reduction surgery before and after MSC therapy in patients with bilateral upper lobe emphysema (44). This study showed no evidence of increased fibrosis in the MSC-treated lung tissue, nor upregulation of pro-fibrotic genes. MSCtreated lung tissue did show a significant increase in endothelial and T-cell markers, which may suggest improved angiogenesis, growth, and repair in response to MSC therapy.

\section{NEONATAL CLINICAL TRIALS OF MSCs FOR BPD}

The first clinical trial investigating the use of MSCs for BPD was a phase I, single-arm, dose-escalation trial carried out by Won Soon Park and colleagues at Samsung Medical Center in Seoul, Korea (12). Nine premature infants were recruited. Inclusion criteria included: gestational age between 23 and 29 weeks, birth weight $500-1,250 \mathrm{~g}$, and need for ventilator support at 5-14 days of age without respiratory improvement over the previous $24 \mathrm{~h}$. Infants with severe congenital anomalies, pulmonary hypoplasia, sepsis, or grade 3 or 4 intraventricular hemorrhage were excluded. The first three infants were given a single IT dose of 10 million cells $/ \mathrm{kg}$ between 7 and 14 days of age. As no immediate adverse events were noted, the remaining six infants were given 20 million cells $/ \mathrm{kg} / \mathrm{dose}$. Infants were closely monitored for adverse events for 84 days following MSC transplantation including death, anaphylactic shock, and serious adverse events (SAEs) defined by the Consolidated Standards of Reporting Trials (49). The investigators also assessed inflammatory markers and growth factors present in tracheal aspirate samples before and after transplantation as well as longer-term outcomes such as incidence and severity of BPD. In this study, IT administration of MSCs appeared to be well tolerated without short-term complications or adverse events. The authors also report a significant reduction in inflammatory markers IL-6, IL-8, TNF- $\alpha$, and TGF- $\beta$ on day 7 post transplantation compared to pre-transplant levels. In the absence of a control group, however, it is difficult to attribute this to MSC therapy vs. natural progression of disease. Of the nine infants transplanted, three went on to develop moderate BPD. Compared to a historical comparison group, this was a significant reduction in BPD. This should be interpreted with caution, however, given the small sample size and limitations of a historical control (of note, the incidence of BPD in their control group was $72 \%$-much higher than expected based on population data (4)).

Subjects of this phase I trial were followed up at regular intervals for 2 years post transplant to evaluate the long-term safety of IT MSC therapy, as well as long-term effects on growth, respiratory symptoms, and neurodevelopmental outcomes (13). One infant from the initial treatment group died at 6 months of age secondary to Enterobacter cloacae sepsis, which was deemed unrelated to prior treatment with MSCs. Of the eight remaining infants in the treatment group, none were discharged home with supplemental oxygen (compared to $22 \%$ in the historical control group) and chest X-rays obtained between 18 and 24 months corrected gestational age 
Table 1. Clinical trials of mesenchymal stem cells for adult and pediatric pulmonary diseases

\begin{tabular}{|c|c|c|c|c|c|c|c|c|c|c|c|c|}
\hline Disease & $\begin{array}{l}\text { Study } \\
\text { type }\end{array}$ & Age & $n$ & $\begin{array}{l}\text { MSC } \\
\text { source }\end{array}$ & Dose & Frequency & y Delivery & Follow-up & Status & Outcome & Identifier & Principal Investigator/location \\
\hline \multicolumn{13}{|c|}{ Bronchopulmonary dysplasia } \\
\hline BPD & Phase I & $\begin{array}{l}\text { Newborn }(7- \\
14 \text { days) }\end{array}$ & & 9 UCB & $\begin{array}{c}10 \times 10^{6} / \\
\mathrm{kg} \\
20 \times 10^{6} / \\
\mathrm{kg}\end{array}$ & $\begin{array}{l}\text { Single } \\
\text { dose }\end{array}$ & IT & $\begin{array}{l}84 \mathrm{~d} / \\
36 \text { weeks } \\
\text { CGA }\end{array}$ & Completed & No SAEs, $\downarrow$ BPD ${ }^{a}$ & NCT01297205 & $\begin{array}{l}5 \text { Won-Soon Park, MD, PhD } \\
\text { Samsung Medical Center, Korea }\end{array}$ \\
\hline BPD & $\begin{array}{l}\text { Phase 1- } \\
\text { follow-up }\end{array}$ & $\begin{array}{l}\text { Newborn }(7- \\
14 \text { days })\end{array}$ & & $9 \quad$ UCB & $\begin{array}{c}10 \times 10^{6} \% \\
\mathrm{~kg} \\
20 \times 10^{6} \% \\
\mathrm{~kg}\end{array}$ & $\begin{array}{l}\text { Single } \\
\text { dose }\end{array}$ & IT & 2 years & Completed & $\begin{array}{l}\text { No sequelae, } \uparrow \\
\text { growth }\end{array}$ & NCT01632475 & $\begin{array}{l}5 \text { Won-Soon Park, PhD } \\
\text { Samsung Medical Center, Korea }\end{array}$ \\
\hline BPD & $\begin{array}{l}\text { Phase I - } \\
\text { follow-up }\end{array}$ & $\begin{array}{c}\text { Newborn (7- } \\
14 \text { days) }\end{array}$ & 9 & 9 UCB & $\begin{array}{c}10 \times 10^{6} \% \\
\mathrm{~kg} \\
20 \times 10^{6} \% \\
\mathrm{~kg}\end{array}$ & $\begin{array}{l}\text { Single } \\
\text { dose }\end{array}$ & IT & 5 years & $\begin{array}{l}\text { Active, not } \\
\text { recruiting }\end{array}$ & N/A & NCT02023788 & $\begin{array}{l}8 \text { Won-Soon Park, PhD } \\
\text { Samsung Medical Center, Korea }\end{array}$ \\
\hline BPD & Phase II & $\begin{array}{l}\text { Newborn (7- } \\
14 \text { days) }\end{array}$ & 70 & $0 \quad$ UCB & $\begin{array}{c}10 \times 10^{6} / \\
\mathrm{kg}\end{array}$ & $\begin{array}{l}\text { Single } \\
\text { dose }\end{array}$ & IT & $\begin{array}{l}84 \mathrm{~d} / \\
36 \text { weeks } \\
\text { CGA }\end{array}$ & Unknown & N/A & NCT01828957 & $\begin{array}{l}7 \text { Won-Soon Park, PhD } \\
\text { Samsung Medical Center, Korea }\end{array}$ \\
\hline BPD & $\begin{array}{l}\text { Phase II- } \\
\text { follow-up }\end{array}$ & $\begin{array}{l}\text { Newborn }(7- \\
14 \text { days })\end{array}$ & 70 & $0 \quad$ UCB & $\begin{array}{c}10 \times 10^{6} / \\
\mathrm{kg}\end{array}$ & $\begin{array}{l}\text { Single } \\
\text { dose }\end{array}$ & IT & 5 years & Recruiting & N/A & NCT01897987 & $\begin{array}{l}7 \text { Won-Soon Park, PhD } \\
\text { Samsung Medical Center, Korea }\end{array}$ \\
\hline BPD & Phase I/II & $\begin{array}{l}\text { Newborn }(7- \\
14 \text { days) }\end{array}$ & 12 & $2 \quad U C B$ & $\begin{array}{c}10 \times 10^{6} / \\
\mathrm{kg} \\
20 \times 10^{6} / \\
\mathrm{kg}\end{array}$ & $\begin{array}{l}\text { Single } \\
\text { dose }\end{array}$ & IT & $\begin{array}{l}84 \mathrm{~d} / 20 \\
\mathrm{mo} \mathrm{CGA}\end{array}$ & $\begin{array}{l}\text { Active, not } \\
\text { recruiting }\end{array}$ & N/A & NCT02381366 & $\begin{array}{l}6 \text { Steven Powell, MD } \\
\text { Rush University Medical Center, USA }\end{array}$ \\
\hline BPD & Phase I & $\begin{array}{l}\text { Infant (1- } \\
6 \text { months) }\end{array}$ & 10 & UC & $\begin{array}{c}3 \times 10^{6} / \\
\mathrm{kg}\end{array}$ & $\begin{array}{l}\text { Single } \\
\text { dose }\end{array}$ & IT & 1 year & Unknown & N/A & NCT01207869 & $\begin{array}{l}9 \text { Bai-Horng Su, MD, PhD } \\
\text { China Medical University Hospital, China }\end{array}$ \\
\hline BPD & Phase I & $\begin{array}{l}\text { Newborn } \\
(>14 \text { days })\end{array}$ & 10 & 0 Unknown & $\begin{array}{c}5 \times 10^{6} / \\
\mathrm{kg}\end{array}$ & $\begin{array}{l}\text { Repeated } \\
\text { doses }\end{array}$ & IT & 2 years & $\begin{array}{l}\text { Not yet } \\
\text { recruiting }\end{array}$ & N/A & NCT02443961 & $\begin{array}{l}1 \text { Maria Jesus del Cerro, PhD } \\
\text { Hospital Universitario Ramón y Cajal, Spain }\end{array}$ \\
\hline \multicolumn{13}{|c|}{ Acute respiratory distress syndrome } \\
\hline ARDS & Phase I & $\begin{array}{l}\text { Adult }(18-90 \\
\text { years })\end{array}$ & 20 & 0 Adipose & $\begin{array}{c}1 \times 10^{6} / \\
\mathrm{kg}\end{array}$ & $\begin{array}{l}\text { Single } \\
\text { dose }\end{array}$ & IV & $1 \mathrm{mo}$ & Unknown & $\mathrm{N} / \mathrm{A}$ & NCT01902082 & $\begin{array}{l}2 \text { Jianguo } \mathrm{Xu}, \mathrm{PhD} \\
\text { Shaoxing Second Hospital, China }\end{array}$ \\
\hline ARDS & Phase I/II & $\begin{array}{l}\text { Adult }(35-70 \\
\text { years) }\end{array}$ & 20 & UC & Unknown & $\begin{array}{l}\text { Single } \\
\text { dose }\end{array}$ & IV & 2 weeks & Recruiting & N/A & NCT02444455 & $\begin{array}{l}5 \text { Huiying Liu, MD } \\
\text { Affiliated Hospital to Academy of Military } \\
\text { Medical Sciences, China }\end{array}$ \\
\hline ARDS & Phase I & $\begin{array}{l}\text { Adult }(>18 \\
\quad \text { years })\end{array}$ & 9 & BM & $\begin{array}{c}1 \times 10^{6} \% \\
\mathrm{~kg} \\
5 \times 10^{6} \% \\
\mathrm{~kg} \\
10 \times 10^{6} \% \\
\mathrm{~kg}\end{array}$ & $\begin{array}{l}\text { Single } \\
\text { dose }\end{array}$ & IV & 1 year & Completed & No SAEs & NCT01775774 & $\begin{array}{l}4 \text { Michael Matthay, MD } \\
\text { University of California, San Francisco, USA }\end{array}$ \\
\hline ARDS & Phase II & $\begin{array}{l}\text { Adult }(>18 \\
\text { years })\end{array}$ & 60 & BM & $\begin{array}{c}10 \times 10^{6} / \\
\mathrm{kg}\end{array}$ & $\begin{array}{l}\text { Single } \\
\text { dose }\end{array}$ & IV & 1 year & Recruiting & N/A & NCT02097641 & $\begin{array}{l}1 \text { Michael Matthay, MD } \\
\text { University of California, San Francisco, USA }\end{array}$ \\
\hline ARDS & Phase II & & 10 & BM & Unknown & & IV & $1 \mathrm{mo}$ & Recruiting & $\mathrm{N} / \mathrm{A}$ & NCT02112500 & \\
\hline
\end{tabular}


Table 1 Continued

\begin{tabular}{|c|c|c|c|c|c|c|c|c|c|c|c|c|}
\hline \multirow[t]{2}{*}{ Disease } & \multirow[t]{2}{*}{$\begin{array}{l}\text { Study } \\
\text { type }\end{array}$} & Age & \multirow[t]{2}{*}{$n$} & $\begin{array}{l}\text { MSC } \\
\text { source }\end{array}$ & \multirow[t]{2}{*}{ Dose } & \multicolumn{2}{|c|}{ Frequency Delivery } & \multirow[t]{2}{*}{ Follow-up } & \multirow[t]{2}{*}{ Status } & \multirow[t]{2}{*}{ Outcome } & \multirow[t]{2}{*}{ Identifier } & \multirow{2}{*}{$\begin{array}{l}\text { Principal Investigator/location } \\
\text { Sang Bum Hong, MD } \\
\text { Asan Medical Center, Korea }\end{array}$} \\
\hline & & $\begin{array}{l}\text { Adult }(20-80 \\
\quad \text { years })\end{array}$ & & & & $\begin{array}{l}\text { Single } \\
\text { dose }\end{array}$ & & & & & & \\
\hline ARDS & Phase I/II & $\begin{array}{l}\text { Adolescent/ } \\
\text { adult }(>16 \\
\text { years) }\end{array}$ & 75 & UC & Unknown & $\begin{array}{l}\text { Single } \\
\text { dose }\end{array}$ & IV & $1 \mathrm{mo}$ & $\begin{array}{l}\text { Not yet } \\
\text { recruiting }\end{array}$ & N/A & NCT0304214 & $\begin{array}{l}3 \text { Danny McAuley, MD } \\
\text { Belfast Health and Social Care Trust, Ireland }\end{array}$ \\
\hline ARDS after BMT & Phase II & $\begin{array}{l}\text { Adult }(>18 \\
\quad \text { years })\end{array}$ & 50 & Unknown & $\begin{array}{c}3 \times 10^{6} / \\
\mathrm{kg}\end{array}$ & $\begin{array}{l}\text { Single } \\
\text { dose }\end{array}$ & IV & $1 \mathrm{mo}$ & Recruiting & N/A & NCT0280494 & $\begin{array}{l}5 \text { Amanda Olson, MD } \\
\text { University of Texas MD Anderson Cancer } \\
\text { Center, USA }\end{array}$ \\
\hline \multicolumn{13}{|c|}{ Obstructive lung diseases } \\
\hline Emphysema & Phase I & $\begin{array}{l}\text { Adolescent/ } \\
\text { adult }(16-70 \\
\text { years })\end{array}$ & 12 & BM & $60 \times 10^{6}$ & $\begin{array}{l}\text { Single } \\
\text { dose }\end{array}$ & IT & 1 year & Unknown & $\mathrm{N} / \mathrm{A}$ & NCT0175805 & $\begin{array}{l}5 \text { Paritash Tahmaseb Pour, MD, Hoda Madani, } \\
\text { MD } \\
\text { Masih-Daneshvari Hospital, Iran }\end{array}$ \\
\hline Emphysema & Phase I & $\begin{array}{l}\text { Adult }(40-65 \\
\text { years) }\end{array}$ & 10 & BM & Unknown & $\begin{array}{l}\text { Single } \\
\text { dose }\end{array}$ & IV & 1 year & Completed & $\begin{array}{l}\text { No SAEs, no fibrosis } \\
\text { or tumor formation }\end{array}$ & NCT0130651 & $\begin{array}{l}3 \text { Jan Stolk, MD, PhD } \\
\text { Leiden University Medical Center, The } \\
\text { Netherlands }\end{array}$ \\
\hline Emphysema & Phase I/II & $\begin{array}{l}\text { Adult }(35-75 \\
\text { years })\end{array}$ & 30 & BM & $200 \times 10^{6}$ & $\begin{array}{l}\text { Repeated } \\
\text { doses }\end{array}$ & IV & 2 years & Recruiting & N/A & NCT0184915 & $\begin{array}{l}9 \text { Alexander V Averyanov, MD, PhD } \\
\text { Federal Research Clinical Center of Federal } \\
\text { Medical and Biological Agency, Russia }\end{array}$ \\
\hline COPD & Phase I/II & $\begin{array}{l}\text { Adult }(40-80 \\
\text { years) }\end{array}$ & 20 & Adipose & Unknown & $\begin{array}{l}\text { Single } \\
\text { dose }\end{array}$ & IV & 1 year & Recruiting & N/A & NCT0264530 & $\begin{array}{l}5 \text { Phuong Le Thi Bich, MSc, MD, Chien Vo } \\
\text { Duc, MD } \\
\text { Van Hanh Hospital, Vietnam }\end{array}$ \\
\hline COPD & Phase II & $\begin{array}{l}\text { Adult }(40-80 \\
\text { years) }\end{array}$ & 62 & BM & $100 \times 10^{6}$ & $\begin{array}{l}\text { Repeated } \\
\text { doses }\end{array}$ & IV & 2 years & Completed & No SAEs, $\downarrow$ CRP & NCT0068372 & $\begin{array}{l}2 \text { Mesoblast International Sàrl—Multiple Sites } \\
\text { USA }\end{array}$ \\
\hline Bronchiectasis & Phase I & $\begin{array}{l}\text { Adult }(30-87 \\
\quad \text { years })\end{array}$ & 6 & BM & $\begin{array}{c}20 \times 10^{6} \\
100 \times 10^{6}\end{array}$ & $\begin{array}{l}\text { Single } \\
\text { dose }\end{array}$ & IV & 1 year & Recruiting & N/A & NCT0262524 & $\begin{array}{r}6 \text { Marilyn K Glassberg, MD } \\
\text { University of Miami, USA }\end{array}$ \\
\hline \multicolumn{13}{|c|}{ Restrictive lung diseases } \\
\hline IPF & Phase I & $\begin{array}{l}\text { Adult }(40-80 \\
\quad \text { years })\end{array}$ & 8 & Placenta & $\begin{array}{c}1 \times 10^{6} / \\
\mathrm{kg} \\
2 \times 10^{6} / \\
\mathrm{kg}\end{array}$ & $\begin{array}{l}\text { Single } \\
\text { dose }\end{array}$ & IV & 6 months & Completed & $\begin{array}{l}\text { No SAEs, no tumor } \\
\text { formation }\end{array}$ & NCT0138564 & $\begin{array}{l}4 \text { Daniel Chambers, MBBS, MD } \\
\text { The Prince Charles Hospital Australia }\end{array}$ \\
\hline IPF & Phase I & $\begin{array}{l}\text { Adult }(30-80 \\
\text { years })\end{array}$ & 17 & BM & Unknown & $\begin{array}{l}\text { Single } \\
\text { dose }\end{array}$ & IV & 1 year & $\begin{array}{l}\text { Active, not } \\
\text { recruiting }\end{array}$ & N/A & NCT0191982 & 7 Clinica Universidad de Navarra, Spain \\
\hline IPF & Phase I & $\begin{array}{l}\text { Adult }(40-90 \\
\text { years) }\end{array}$ & 25 & Unknown & $\begin{array}{c}2 \times 10^{6} \\
100 \times 10^{6} \\
200 \times 10^{6}\end{array}$ & $\begin{array}{l}\text { Single } \\
\text { dose }\end{array}$ & IV & 60 wks & $\begin{array}{l}\text { Active, not } \\
\text { recruiting }\end{array}$ & N/A & NCT0201370 & $\begin{array}{l}\text { O Marilyn K. Glassberg, MD } \\
\text { University of Miami, USA }\end{array}$ \\
\hline $\begin{array}{l}\text { Radiation- } \\
\text { induced PF }\end{array}$ & Phase I & $\begin{array}{l}\text { Adult }(18-70 \\
\text { years })\end{array}$ & 14 & UC & $\begin{array}{c}1 \times 10^{6} / \\
\mathrm{kg}\end{array}$ & $\begin{array}{l}\text { Single } \\
\text { dose }\end{array}$ & IT & 6 months & $\begin{array}{l}\text { Active, not } \\
\text { recruiting }\end{array}$ & N/A & NCT0227714 & $\begin{array}{l}5 \text { JianWu Dai, Ph.D } \\
\text { Chinese Academy of Sciences, China }\end{array}$ \\
\hline Pneumoconiosis & Phase I & $\begin{array}{l}\text { Adult }(18-70 \\
\text { years) }\end{array}$ & 80 & UC & $\begin{array}{c}1 \times 10^{6} / \\
\mathrm{kg}\end{array}$ & $\begin{array}{l}\text { Single } \\
\text { dose }\end{array}$ & IT & 6 months & Recruiting & $\mathrm{N} / \mathrm{A}$ & NCT0266806 & $\begin{array}{l}8 \text { JianWu Dai, Ph.D } \\
\text { Chinese Academy of Sciences, China }\end{array}$ \\
\hline
\end{tabular}




\begin{tabular}{|c|c|c|c|c|c|c|c|c|c|c|c|c|}
\hline Disease & $\begin{array}{l}\text { Study } \\
\text { type }\end{array}$ & Age & $n$ & $\begin{array}{l}\text { MSC } \\
\text { source }\end{array}$ & Dose & Frequency & yelivery & Follow-up & Status & Outcome & Identifier & Principal Investigator/location \\
\hline Pneumoconiosis & Phase I & $\begin{array}{l}\text { Adult }(18-75 \\
\quad \text { years })\end{array}$ & 10 & UC & $\begin{array}{c}20 \times 10^{6} \% \\
\mathrm{~kg}\end{array}$ & $\begin{array}{l}\text { Repeated } \\
\text { doses }\end{array}$ & IV & 1 year & Recruiting & $\mathrm{N} / \mathrm{A}$ & NCT02790762 & $\begin{array}{l}2 \text { Guo Q Li, Professor } \\
\text { The Second Affiliated Hospital of University } \\
\text { of South China, China }\end{array}$ \\
\hline $\begin{array}{l}\text { Pulmonary } \\
\text { hemonthsiderosis }\end{array}$ & Phase I & $\begin{array}{l}\text { Child }(1-18 \\
\text { years) }\end{array}$ & 100 & BM & Unknown & Unknown & Unknown & 6 months & $\begin{array}{l}\text { Not yet } \\
\text { recruiting }\end{array}$ & $\mathrm{N} / \mathrm{A}$ & NCT02985346 & $\begin{array}{l}6 \text { Weiping Tan } \\
\text { Sun Yat-sen Memorial Hospital, China }\end{array}$ \\
\hline ILD & Phase I/II & $\begin{array}{l}\text { Adult }(20-80 \\
\quad \text { years })\end{array}$ & 20 & BM & $200 \times 10^{6}$ & $\begin{array}{l}\text { Repeated } \\
\text { doses }\end{array}$ & IV & 1 year & Recruiting & $\mathrm{N} / \mathrm{A}$ & NCT02594839 & $\begin{array}{l}9 \text { Alexander Averyanov, MD } \\
\text { Federal Research Clinical Center of Federal } \\
\text { Medical and Biological Agency, Russia }\end{array}$ \\
\hline IPF & Phase I/II & $\begin{array}{l}\text { Adult }(30-70 \\
\text { years) }\end{array}$ & 60 & Adipose & $\begin{array}{c}2 \times 10^{6} / \\
\mathrm{kg}\end{array}$ & $\begin{array}{l}\text { Repeated } \\
\text { doses }\end{array}$ & IV & 9 months & Unknown & $\mathrm{N} / \mathrm{A}$ & NCT02135380 & $\begin{array}{l}0 \text { Ashok A Mahashur, MBBS, MD et. al. } \\
\text { Multiple centers, India }\end{array}$ \\
\hline ILD after BMT & Phase I/II & $\begin{array}{l}\text { Adult }(18-65 \\
\quad \text { years })\end{array}$ & & Unknown & $\begin{array}{c}1 \times 10^{6} / \\
\mathrm{kg}\end{array}$ & $\begin{array}{l}\text { Repeated } \\
\text { doses }\end{array}$ & Unknown & 3 years & $\begin{array}{l}\text { Active, not } \\
\text { recruiting }\end{array}$ & $N / A$ & NCT02543073 & $\begin{array}{l}3 \text { Qifa Liu, MD } \\
\text { Nanfang Hospital of Southern Medical } \\
\text { University, China }\end{array}$ \\
\hline \multicolumn{13}{|l|}{ Cystic fibrosis } \\
\hline Cystic fibrosis & Phase I & $\begin{array}{l}\text { Adult }(18-40 \\
\quad \text { years })\end{array}$ & 15 & BM & $\begin{array}{c}1 \times 10^{6} / \\
\mathrm{kg} \\
3 \times 10^{6} / \\
\mathrm{kg} \\
5 \times 10^{6} \% \\
\mathrm{~kg}\end{array}$ & $\begin{array}{l}\text { Single } \\
\text { dose }\end{array}$ & IV & 1 year & Recruiting & $\mathrm{N} / \mathrm{A}$ & NCT02866721 & $\begin{array}{l}1 \text { James Chmiel, MD } \\
\text { University Hospitals Cleveland Medical } \\
\text { Center, USA }\end{array}$ \\
\hline Cystic fibrosis & Phase I & $\begin{array}{l}\text { Adult }(20-45 \\
\quad \text { years })\end{array}$ & & 3 Unknown & $\begin{array}{r}20 \times 10^{6} \\
100 \times 10^{6}\end{array}$ & $\begin{array}{l}\text { Single } \\
\text { dose }\end{array}$ & IV & 1 year & $\begin{array}{l}\text { Not yet } \\
\text { recruiting }\end{array}$ & $\mathrm{N} / \mathrm{A}$ & NCT03058068 & $\begin{array}{l}8 \text { Matthias Salathe, MD } \\
\text { University of Miami, USA }\end{array}$ \\
\hline \multicolumn{13}{|c|}{ Lung transplant/surgical } \\
\hline $\begin{array}{l}\text { Lung transplant } \\
\text { rejection/BO }\end{array}$ & Phase I & $\begin{array}{l}\text { Adult }(18-75 \\
\quad \text { years })\end{array}$ & & Unknown & $\begin{array}{c}2- \\
4 \times 10^{6} / \\
\mathrm{kg}\end{array}$ & $\begin{array}{l}\text { Single } \\
\text { dose }\end{array}$ & IV & 2 weeks & $\begin{array}{l}\text { Active, not } \\
\text { recruiting }\end{array}$ & $N / A$ & NCT02181712 & $\begin{array}{l}2 \text { Cesar Keller, MD, Abba C Zubair, MD, PhD } \\
\text { Mayo Clinic, USA }\end{array}$ \\
\hline Lung resection & Phase I/II & $\begin{array}{l}\text { Adult }(18-70 \\
\quad \text { years })\end{array}$ & 10 & BM & Unknown & $\begin{array}{l}\text { Single } \\
\text { dose }\end{array}$ & Local & 2 years & Recruiting & $\mathrm{N} / \mathrm{A}$ & NCT02045745 & $\begin{array}{l}5 \text { Marcelo F Jiménez López, PhD } \\
\text { University Clinical Hospital of Salamanca, } \\
\text { Spain }\end{array}$ \\
\hline \multicolumn{13}{|c|}{ Toxin-induced lung injury } \\
\hline Mustard lung & Phase I & $\begin{array}{l}\text { Adult }(45-65 \\
\quad \text { years })\end{array}$ & 10 & BM & $100 \times 10^{6}$ & $\begin{array}{l}\text { Repeated } \\
\text { doses }\end{array}$ & IV & 6 months & $\begin{array}{l}\text { Active, not } \\
\text { recruiting }\end{array}$ & N/A & NCT02749448 & $\begin{array}{l}8 \text { Mostafa Ghanei, MD } \\
\text { Baqiyatallah Medical Sciences University, } \\
\text { Iran }\end{array}$ \\
\hline $\begin{array}{l}\text { Paraquat } \\
\text { poisoning- } \\
\text { induced LI }\end{array}$ & Phase I/II & $\begin{array}{l}\text { Adolescent/ } \\
\text { adult (15-60 } \\
\text { years) }\end{array}$ & 40 & UC & $0.5 \times 10^{6}$ & $\begin{array}{l}\text { Repeated } \\
\text { doses }\end{array}$ & IV & 2 months & Recruiting & $\mathrm{N} / \mathrm{A}$ & NCT02444858 & $\begin{array}{l}8 \text { Haochun Wang, MD et. al. } \\
\text { Affiliated Hospital to Academy of Military } \\
\text { Medical Sciences, China }\end{array}$ \\
\hline
\end{tabular}


(CGA) showed no evidence of mass lesion or other abnormality. Body weight was significantly higher in the transplantation group at 18-24 months CGA. Length and head circumference were not different. There were no significant differences in rates of neurologic sequelae (cerebral palsy, seizures, and blindness), nor neurodevelopmental outcomes based on assessment with the Bayley Scales of Infant Developmental-Second Edition, cognitive adaptive scale, and clinical linguistic and auditory milestone scale at 18-24 months CGA. Brain MRIs obtained at two time-points (36-40 weeks and 18-24 months CGA) showed no changes that could be attributed to MSC treatment. These findings support the safety of intra-tracheal MSCs up to 24 months CGA in preterm infants. The investigators are planning longterm follow-up of these subjects to 5 years of age (50). Decreased need for supplemental oxygen at discharge compared to historical controls is encouraging that IT MSCs may be an effective therapy for BPD, however larger studies are needed to provide more evidence of the safety and efficacy of this therapy. The investigators of this study are currently recruiting for a randomized, double-blind, placebo-controlled phase II study with long-term follow-up $(51,52)$. In this phase II study, a single IT dose of $1 \times 10^{7}$ cells $/ \mathrm{kg}$ will be administered to premature infants at risk for BPD between 7 and 14 days of age. Subjects will be followed up to 5 years of age with a target recruitment of 70 subjects.

Multiple additional trials investigating the use of MSCs for BPD are ongoing in the United States, Spain, and China. The first US study is a phase I/II dose escalation trial of allogenic umbilical cord-derived MSCs (53) with similar criteria and outcomes to the Korean trial. Twelve neonates with a mean gestational age of 24.9 weeks and mean birth weight of $676 \mathrm{~g}$ have been recruited for this study. Infants received a single IT dose of either $1 \times 10^{7}$ or $2 \times 10^{7}$ cells/kg at $10.6 \pm 2.9$ days of life. Results of this study have not yet been published, however preliminary reports indicate that MSC administration was well-tolerated without short-term cardio-respiratory compromise. There were no deaths or SAEs directly attributable to MSC treatment, however there was one late death of a study subject at 116 days secondary to pulmonary hypertension and pulmonary hypoplasia (54). A phase I study in Spain will be investigating the safety of three serial doses ( 5 million cells/ $\mathrm{kg} /$ dose) of IT MSCs (55). They are not yet recruiting, but plan to study 10 infants $<28$ weeks gestation and $<1,250 \mathrm{~g}$ requiring mechanical ventilation and $\mathrm{FiO}_{2}>0.3$ at 14 days of age. They plan to follow infant outcomes for 2 years with specific interest in the incidence and severity of BPD and pulmonary hypertension. In China, the safety of IT MSCs will be investigated as a rescue therapy for infants $<6$ months of age with established, severe BPD (56). A single dose of 3 million cells/kg will be administered intratracheally. Cytokine levels in bronchoalveolar lavage fluid, chest radiographs, and clinical outcomes will be followed. Target recruitment is 10 subjects; however, recruitment status is currently unknown and no results have been published to date.

\section{BARRIERS TO MSC THERAPY FOR CLINICAL USE}

Even if clinical trials of MSCs for BPD prove successful, there remain significant challenges to translating this therapy into clinical use. One primary factor complicating the selection of MSCs for transplant is the heterogeneity of MSC populations (57). MSCs have variable phenotypes-and thus differing effectiveness-depending on the donor and tissue type from which they are harvested. MSCs derived from gestational tissue (umbilical cord blood, Wharton's jelly, and placenta) have been found to be less antigenic, have higher proliferative capacity, and have stronger paracrine potency when compared to bone marrow-derived MSCs (58). Interestingly, MSCs derived from females secrete more anti-inflammatory and pro-angiogenic factors compared to MSCs derived from males, and in animal models, MSCs derived from females exhibited greater efficacy in reducing neonatal hyperoxiainduced lung disease compared to MSCs derived from males (59).

Another hurdle to overcome is the logistical challenge of large-scale production of MSCs for transplant. Commercial preparation of MSCs relies on extensive ex vivo expansion and cryopreservation to produce large quantities of readily available cells. Ex vivo growth of MSCs is limited by senescence (16), however, and heavily expanded MSCs may be less effective due to the high percentage of senescent cells. Cryopreservation is also known to impair the immunomodulatory properties of MSCs (60). This may explain why clinical trials investigating the use of MSCs for graft vs. host disease showed better survival outcomes with the use of early passage MSCs compared to commercial, heavily expanded, cryopreserved MSCs $(61,62)$.

For cell-free preparations, as for MSCs themselves, there remains the issue of defining the most efficacious progenitor cell population from which to derive these products (20). For exosomes/extracellular vesicles, there remains a need to define uniform definitions and isolation parameters (i.e., size, surface markers, isolation procedure, and so on) to allow for consistent pre-clinical data generation across investigators $(28,63)$. Finally, for both conditioned media and exosomes, pre-clinical dose and timing experiments are needed to define parameters that would be used in early phase clinical trials. For these reasons, cell-free preparations remain a pre-clinical technology, despite their great clinical potential.

\section{CONCLUSIONS}

Cell-based therapies may prove to be a breakthrough in the prevention and treatment of BPD. Optimism for pursuing additional clinical trials investigating MSCs in BPD is supported by extensive preclinical evidence as well as metaanalysis of MSC trials in older children and adults that show no evidence of adverse events or tumor formation up to 60 months post treatment (40). However, there remain significant logistical barriers as well as gaps in knowledge of the role of endogenous MSCs in the dysregulated repair and pathogenesis of BPD. Thus, concurrent high-quality basic science, translational, and clinical studies investigating the 


\section{Review $\mid$ simones et al.}

fundamental pathophysiology underlying BPD, therapeutic mechanisms of exogenous MSCs, and logistical details of translating cellular therapies into clinical use will be important areas of future research.

Disclosure: The authors declare no conflict of interest.

\section{REFERENCES}

1. Northway WH, Rosan RC, Porter DY. Pulmonary disease following respirator therapy of hyaline-membrane disease. Bronchopulmonary dysplasia. N Engl J Med 1967;276:357-68.

2. Jobe AH, Bancalari E. Bronchopulmonary dysplasia. Am J Respir Crit Care Med 2001;163:1723-9.

3. Bancalari E, Claure N, Sosenko IR. Bronchopulmonary dysplasia: changes in pathogenesis, epidemiology and definition. Semin Neonatol 2003;8: 63-71.

4. Stoll BJ, Hansen NI, Bell EF, et al. Trends in care practices, morbidity, and mortality of extremely preterm neonates, 1993-2012. JAMA 2015;314:1039-51.

5. Jensen EA, Foglia EE, Schmidt B. Evidence-based pharmacologic therapies for prevention of bronchopulmonary dysplasia: application of the grading of recommendations assessment, development, and evaluation methodology. Clin Perinatol 2015;42:755-79.

6. Collins JJ, Thébaud B. Lung mesenchymal stromal cells in development and disease: to serve and protect? Antioxid Redox Signal 2014;21: 1849-62.

7. Chang YS, Oh W, Choi SJ, et al. Human umbilical cord blood-derived mesenchymal stem cells attenuate hyperoxia-induced lung injury in neonatal rats. Cell Transplant 2009;18:869-86.

8. Aslam M, Baveja R, Liang OD, et al. Bone marrow stromal cells attenuate lung injury in a murine model of neonatal chronic lung disease. Am J Respir Crit Care Med 2009;180:1122-30.

9. van Haaften T, Byrne R, Bonnet S, et al. Airway delivery of mesenchymal stem cells prevents arrested alveolar growth in neonatal lung injury in rats. Am J Respir Crit Care Med 2009;180:1131-42.

10. Chang YS, Choi SJ, Ahn SY, et al. Timing of umbilical cord blood derived mesenchymal stem cells transplantation determines therapeutic efficacy in the neonatal hyperoxic lung injury. PLoS One 2013;8:e52419.

11. Chang YS, Choi SJ, Sung DK, et al. Intratracheal transplantation of human umbilical cord blood-derived mesenchymal stem cells dosedependently attenuates hyperoxia-induced lung injury in neonatal rats. Cell Transplant 2011;20:1843-54.

12. Chang YS, Ahn SY, Yoo HS, et al. Mesenchymal stem cells for bronchopulmonary dysplasia: phase 1 dose-escalation clinical trial. J Pediatr 2014;164:966-972.e966.

13. Ahn SY, Chang YS, Kim JH, Sung SI, Park WS. Two-year follow-up outcomes of premature infants enrolled in the phase I trial of mesenchymal stem cells transplantation for bronchopulmonary dysplasia. J Pediatr 2017;185:49-54.e2.

14. Waterman RS, Tomchuck SL, Henkle SL, Betancourt AM. A new mesenchymal stem cell (MSC) paradigm: polarization into a proinflammatory MSC1 or an Immunosuppressive MSC2 phenotype. PLoS ONE 2010;5:e10088.

15. da Silva Meirelles L, Chagastelles PC, Nardi NB. Mesenchymal stem cells reside in virtually all post-natal organs and tissues. J Cell Sci 2006;119: 2204-13.

16. Dominici M, Le Blanc K, Mueller I, et al. Minimal criteria for defining multipotent mesenchymal stromal cells. The International Society for Cellular Therapy position statement. Cytotherapy 2006;8:315-7.

17. Pierro M, Ionescu L, Montemurro T, et al. Short-term, long-term and paracrine effect of human umbilical cord-derived stem cells in lung injury prevention and repair in experimental bronchopulmonary dysplasia. Thorax 2013;68:475-84.
18. Ionescu L, Byrne RN, van Haaften T, et al. Stem cell conditioned medium improves acute lung injury in mice: in vivo evidence for stem cell paracrine action. Am J Physiol Lung Cell Mol Physiol 2012;303:L967-977.

19. Tropea KA, Leder E, Aslam M, et al. Bronchioalveolar stem cells increase after mesenchymal stromal cell treatment in a mouse model of bronchopulmonary dysplasia. Am J Physiol Lung Cell Mol Physiol 2012;302:L829-837.

20. Fung ME, Thébaud B. Stem cell-based therapy for neonatal lung disease: it is in the juice. Pediatr Res 2014;75:2-7.

21. Sdrimas K, Kourembanas S. MSC microvesicles for the treatment of lung disease: a new paradigm for cell-free therapy. Antioxid Redox Signal 2014;21:1905-15.

22. Baglio SR, Pegtel DM, Baldini N. Mesenchymal stem cell secreted vesicles provide novel opportunities in (stem) cell-free therapy. Front Physiol 2012;3:359.

23. Gazdic M, Volarevic V, Arsenijevic N, Stojkovic M. Mesenchymal stem cells: a friend or foe in immune-mediated diseases. Stem Cell Rev 2015;11: 280-7.

24. Chen J, Li C, Chen L. The role of microvesicles derived from mesenchymal stem cells in lung diseases. Biomed Res Int 2015;2015:985814.

25. Jaimes Y, Naaldijk Y, Wenk K, Leovsky C, Emmrich F. Mesenchymal stem cell-derived microvesicles modulate lipopolysaccharides-induced inflammatory responses to microglia cells. Stem Cells 2017;35:812-23.

26. Chen JY, An R, Liu ZJ, et al. Therapeutic effects of mesenchymal stem cell-derived microvesicles on pulmonary arterial hypertension in rats. Acta Pharmacol Sin 2014;35:1121-8.

27. Guo H, Cyr-Depauw C, Vadivel A, Zhong S, Thebaud B Therapeutic Potential Human Umbilical Mesenchymal Stromal Cell (MSC)-Derived Exosomes in Experimental Bronchopulmonary Dysplasia. Pediatric Academic Societies Meeting, San Francisco, CA, 2017.

28. Kubo H. Extracellular vesicles in lung disease. Chest 2017.

29. Sinclair K, Yerkovich ST, Chambers DC. Mesenchymal stem cells and the lung. Respirology 2013;18:397-411.

30. Möbius MA, Thébaud B. Bronchopulmonary dysplasia-where have all the stem cells gone? Origin and (potential) function of resident lung stem cells. Chest 2017.

31. Park JS, Chu JS, Tsou AD, et al. The effect of matrix stiffness on the differentiation of mesenchymal stem cells in response to TGF- $\beta$. Biomaterials 2011;32:3921-30.

32. Popova AP, Bozyk PD, Goldsmith AM, et al. Autocrine production of TGF-betal promotes myofibroblastic differentiation of neonatal lung mesenchymal stem cells. Am J Physiol Lung Cell Mol Physiol 2010;298: L735-743.

33. Ling L, Nurcombe V, Cool SM. Wnt signaling controls the fate of mesenchymal stem cells. Gene 2009;433:1-7.

34. Ng F, Boucher S, Koh S, et al. PDGF, TGF-beta, and FGF signaling is important for differentiation and growth of mesenchymal stem cells (MSCs): transcriptional profiling can identify markers and signaling pathways important in differentiation of MSCs into adipogenic, chondrogenic, and osteogenic lineages. Blood 2008;112:295-307.

35. Popova AP, Bozyk PD, Bentley JK, et al. Isolation of tracheal aspirate mesenchymal stromal cells predicts bronchopulmonary dysplasia. Pediatrics 2010;126:e1127-1133.

36. Vento G, Capoluongo E, Matassa PG, et al. Serum levels of seven cytokines in premature ventilated newborns: correlations with old and new forms of bronchopulmonary dysplasia. Intensive Care Med 2006;32: 723-30.

37. Kotecha S, Wangoo A, Silverman M, Shaw RJ. Increase in the concentration of transforming growth factor beta-1 in bronchoalveolar lavage fluid before development of chronic lung disease of prematurity. J Pediatr 1996;128:464-9.

38. Bozyk PD, Popova AP, Bentley JK, et al. Mesenchymal stromal cells from neonatal tracheal aspirates demonstrate a pattern of lung-specific gene expression. Stem Cells Dev 2011;20:1995-2007.

39. Raza K, Larsen T, Samaratunga N, et al. MSC therapy attenuates obliterative bronchiolitis after murine bone marrow transplant. PLoS ONE 2014;9:e109034. 


\section{MSCs for BPD: a clinical review Review}

40. Lalu MM, McIntyre L, Pugliese C, et al. Safety of cell therapy with mesenchymal stromal cells (SafeCell): a systematic review and metaanalysis of clinical trials. PLoS ONE 2012;7:e47559.

41. Duijvestein M, Vos AC, Roelofs $\mathrm{H}$, et al. Autologous bone marrowderived mesenchymal stromal cell treatment for refractory luminal Crohn's disease: results of a phase I study. Gut 2010;59:1662-9.

42. https://www.clinicaltrials.gov/ct2/results?term=mesenchymal+stem + cell\&Search=Search. Accessed 18 August 2017.

43. Weiss DJ, Casaburi R, Flannery R, LeRoux-Williams M, Tashkin DP. A placebo-controlled, randomized trial of mesenchymal stem cells in COPD. Chest 2013;143:1590-8.

44. Stolk J, Broekman W, Mauad T, et al. A phase I study for intravenous autologous mesenchymal stromal cell administration to patients with severe emphysema. QJM 2016;109:331-6.

45. Zheng G, Huang L, Tong H, et al. Treatment of acute respiratory distress syndrome with allogeneic adipose-derived mesenchymal stem cells: a randomized, placebo-controlled pilot study. Respir Res 2014;15:39.

46. Wilson JG, Liu KD, Zhuo H, et al. Mesenchymal stem (stromal) cells for treatment of ARDS: a phase 1 clinical trial. Lancet Respir Med 2015;3:24-32.

47. Tzouvelekis A, Paspaliaris V, Koliakos G, et al. A prospective, nonrandomized, no placebo-controlled, phase Ib clinical trial to study the safety of the adipose derived stromal cells-stromal vascular fraction in idiopathic pulmonary fibrosis. J Transl Med 2013;11:171.

48. Chambers DC, Enever D, Ilic N, et al. A phase $1 \mathrm{~b}$ study of placentaderived mesenchymal stromal cells in patients with idiopathic pulmonary fibrosis. Respirology 2014;19:1013-8.

49. Schulz KF, Altman DG, Moher DGroup CONSORT. CONSORT 2010 Statement: updated guidelines for reporting parallel group randomised trials. Trials 2010;11:32.

50. Medipost Co Ltd Long-Term Safety and Efficacy Follow-up Study of PNEUMOSTEM $^{\circledast}$ in Patients Who Completed PNEUMOSTEM ${ }^{\circledast}$ Phase-I Study, 2014 https://clinicaltrials.gov/show/NCT02023788) ..

51. Medipost Co Ltd Efficacy and Safety Evaluation of Pneumostem ${ }^{\oplus}$ Versus a Control Group for Treatment of BPD in Premature Infants, 2013 https://clinicaltrials.gov/show/NCT01828957) ..

52. Medipost Co Ltd Follow-up Safety and Efficacy Evaluation on Subjects Who Completed PNEUMOSTEM ${ }^{\oplus}$ Phase-II Clinical Trial, 2014 https:// clinicaltrials.gov/show/NCT01897987) ..

53. Medipost America Inc., Medipost Co Ltd Safety and Efficacy of PNEUMOSTEM $^{\oplus}$ in Premature Infants at High Risk for
Bronchopulmonary Dysplasia (BPD)—a US Study, 2015 https:// clinicaltrials.gov/show/NCT02381366.

54. Powell SB, Silvestri JM Safety of Intratracheal Transplantation of Human Umbilical Cord Derived Mesenchymal Stem Cells (HUCB-MSCs) in Extremely Low Birth Weight (ELBW) Preterm Infants at Highest Risk for Bronchopulmonary Dysplasia (BPD). Pediatric Academic Societies Meeting, San Francisco, CA, 2017.

55. Cajal FplIBdHURy, III IdSC, pulmonar FdAalIslH Mesenchymal Stem Cell Therapy for Bronchopulmonary Dysplasia in Preterm Babies, 2017 https://clinicaltrials.gov/show/NCT02443961.

56. Hospital CMU Intratracheal Umbilical Cord-derived Mesenchymal Stem Cells for Severe Bronchopulmonary Dysplasia, 2010 https://clinicaltrials. gov/show/NCT01207869) ..

57. Morita Y, Ema H, Nakauchi H. Heterogeneity and hierarchy within the most primitive hematopoietic stem cell compartment. J Exp Med 2010;207:1173-82.

58. Amable PR, Teixeira MV, Carias RB, Granjeiro JM, Borojevic R. Protein synthesis and secretion in human mesenchymal cells derived from bone marrow, adipose tissue and Wharton's jelly. Stem Cell Res Ther 2014;5:53.

59. Sammour I, Somashekar S, Huang J, et al. The effect of gender on mesenchymal stem cell (MSC) efficacy in neonatal hyperoxia-induced lung injury. PLoS ONE 2016;11:e0164269.

60. François M, Copland IB, Yuan S, Romieu-Mourez R, Waller EK, Galipeau J. Cryopreserved mesenchymal stromal cells display impaired immunosuppressive properties as a result of heat-shock response and impaired interferon- $\gamma$ licensing. Cytotherapy 2012;14:147-52.

61. Galipeau J. The mesenchymal stromal cells dilemma-does a negative phase III trial of random donor mesenchymal stromal cells in steroidresistant graft-versus-host disease represent a death knell or a bump in the road? Cytotherapy 2013;15:2-8.

62. Le Blanc K, Frassoni F, Ball L, et al. Mesenchymal stem cells for treatment of steroid-resistant, severe, acute graft-versus-host disease: a phase II study. Lancet 2008;371:1579-86.

63. Lötvall J, Hill AF, Hochberg F, et al. Minimal experimental requirements for definition of extracellular vesicles and their functions: a position statement from the International Society for Extracellular Vesicles. J Extracell Vesicles 2014;3:26913.

64. Lane SW, Williams DA, Watt FM. Modulating the stem cell niche for tissue regeneration. Nat Biotechnol 2014;32:795-803. 